\title{
Study of Factors Affecting Medical Incident: 2 Powdered Medication Dispensing
}

\author{
Yuka Miyachi*, Chika Nakayama, Satomi Funahashi, Hiroyuki Shimada, Masayuki Takeuchi, \\ Masafumi Ohnishi, Hiroko Saito, Taeyuki Oshima \\ College of Pharmacy, Kinjo Gakuin University, Nagoya, Japan \\ Email: *y-miyachi@kinjo-u.ac.jp
}

How to cite this paper: Miyachi, Y., Nakayama, C., Funahashi, S., Shimada, H., Takeuchi, M., Ohnishi, M., Saito, H. and Oshima, T. (2019) Study of Factors Affecting Medical Incident: 2 Powdered Medication Dispensing. Pharmacology \& Pharmacy, 10, 309-317.

https://doi.org/10.4236/pp.2019.106025

Received: May 13, 2019

Accepted: June 23, 2019

Published: June 26, 2019

Copyright $\odot 2019$ by author(s) and Scientific Research Publishing Inc. This work is licensed under the Creative Commons Attribution-NonCommercial International License (CC BY-NC 4.0). http://creativecommons.org/licenses/by-nc/4.0/

\begin{abstract}
Medical incidents have been collected, analyzed and built up preventive measures by each medical institution for a long time. For powdered medication, there is the problem that it is difficult to tell at a glance the quantity of the active ingredient in the medication that has been dispensed and the quantities that have been mixed together. Therefore, special prevention measures are considered essential. In this study, we examined the work content of pharmacists' powdered medication dispensing, using an eye-tracking technology of measuring a human eye movement, and studied on factors that affect medical incident. Participants were five pharmacists with 8 to 26 years of working experience (expert), and five pharmacists with less than one year of working experience (newcomer). Gaze measurement experiments were implemented for powdered medication dispensing during regular work activity. The gaze measurement equipment used was Tobii Pro Glasses 2. Based on the results of the eye tracking, newcomer had a longer dispensing time than expert for all powdered medication dispensing. In particular, it was suggested that there is a close relationship to "years of experience" and "weighing and mixing skills." Experts did unwasted and efficient movements, when preparing the dispensing apparatus, taking medications from the shelves, and scanning the barcode in the powders dispensing checking system. These movements led to shorter working time in experts. In contrast, newcomer had individual differences at the dispensing. Even with the same pharmacist, the work progression differed depending upon the prescription. Therefore, it is thought that the factor of common error was inadequate check and overlooked. The state that it's messy on the workplace is also considered highly likely to cause dispensing mistakes. At the weighing, expert started weighing after the inspection of the prescription and checking weighed amount. However, certain newcomer dispensed to depend on the powders dispensing checking system only for the weighing process, without the inspection of the
\end{abstract}


prescription or checking weighed amount. Irregular doses for infants and older patients require fine adjustments; therefore, the powders dispensing checking system may not find all dispensing errors. It is important for a pharmacist to, first, be written calculated weight on the prescription and checked by themselves, and next to begin dispensation work. In the future, as well as the powdered medication dispensing, it is necessary to make use of measures for preventing errors in the various dispensing process, such as the medication inspection, sterile products preparation, clinical practice et al.

\section{Keywords}

Dispensing Error, Eye Tracking, Pharmacist, Powdered Medication

\section{Introduction}

Medical incidents (near-miss/adverse event) have been collected, analyzed and built up preventive measures by each medical institution for a long time. 2016 Annual Report of Japan Council for Quality Health Care was reported that approximately $31 \%$ of all accidents was caused by "neglect to check," "neglect to observe" and "misjudgment" [1]. Also, there are "lack of knowledge," "deficiency of technique/skill," "busy working condition" and "under unusual physi$\mathrm{cal} /$ psychological condition" as human factors [2]. Background factors of human error are complex and diverse. Human error won't be a zero, even if there is an excellent prevention measures. The following four stages are important in reducing human error, based on the premise that errors will occur with a certain degree of probability: 1) Reduce dangerous work, 2) Reduce the error rate for each task, 3) Discover errors and resulting action, 4) Prepare to minimize damage [3].

While powdered medication have the advantage that the dosage can be set very precisely, they are easily administered to elderly people and small children, who have difficulty swallowing tablets, and allow multiple medications to be mixed together; however, there is the problem that it is difficult to tell at a glance the quantity of the active ingredient in the medication that has been dispensed and the quantities that have been mixed together [4]. Therefore, special prevention measures are considered essential. Moreover, considering that various rules and internal regulations are set depending on the medical institution, it is thought that the lack of standard lead to dispensing errors. Outline of medical adverse events related to powdered medication are as follows, 1) medication mix-up, 2) dose error, 3) patient misidentification, 4) contamination with another drug. For example, Leukerin was prescribed on the basis of product volume (100 mg), when it should have been prescribed on the basis of active ingredient dosage $(10 \mathrm{mg})$, and $1.5 \mathrm{~g}$ of Atropine Sulfate bulk powder $(\mathrm{g} / \mathrm{g})$ had been dispensed instead of $1.5 \mathrm{mg}$ of Atropine Sulfate powder $(1 \mathrm{mg} / \mathrm{g}$ ) [5].

Evaluating this situation objectively is important to prevent human errors. In 
this study, we focused on an eye-tracking technology. Eye tracking is a tool that provides information on "where, how, and when people look at certain directions," by irradiating the cornea with near infrared light and analyzing images of eye movement [6]. This is a system that enables visualization and measurement of areas of unconscious that cannot be ascertained with words and actions alone. Eye tracking are used in a wide range of applications, including examination of consumer behavior and research on preventive safety of motorists. In past study, we measured for the gaze of the pharmacist and SPD, and examined the factors affecting dispensing error; moreover, we examined prevention of the human error [7].

In this study, we examined the work content of pharmacists' powdered medication dispensing, using an eye-tracking technology of measuring a human eye movement, and studied on factors that affect medical incident.

\section{Method}

\subsection{Subjects}

Subjects were five pharmacists with 1 year more of working experience (expert), and five pharmacists with less than one year of working experience (newcomer). The expert's average working experience was $15.0 \pm 7.2$ years (all female, their age was $30 \mathrm{~s}-40 \mathrm{~s}$ ). The newcomer's working experience was less than a year (3 male and 2 female, their age was $20 \mathrm{~s}-30 \mathrm{~s}$ ). The candidate chose from 78 pharmacists who are working in Aichi Medical University Hospital (Aichi, Japan) at random. This hospital is a general hospital of 900 beds. The investigation was conducted in February of 2018.

\subsection{Measurement Method}

Gaze measurement experiments were implemented for powdered medication dispensing during regular work activity. The gaze measurement equipment used was Tobii Pro Glasses 2 (Tobii Technology K.K. Figure 1). Data on trends of subjects' eye movements, gaze time, and field of vision image data were collected and analyzed. Data were collected when pharmacists would dispense prescriptions that comprised " 1 ) one powder only (ONE-P)," "2) a mixture of two powders (TWO-P)," or "3) a mixture of crushed tablets and lactose (TAB-P)".

The imaging records collected through Tobii Pro Glasses 2 were analyzed using Tobii Pro Lab (Tobii Technology K.K.) and Windows Live Movie Maker. Student's unpaired t-test was used for statistical processing.

\subsection{Gaze Points}

The gaze point of regard within the whole work was divided into 10 regions. The divisions were 1) prescription, 2) medications, 3) inspection system PC (computer screen for the powders dispensing checking system), 4) medications' shelf, 5) weighting scale, 6) dispensing apparatus (pestle and mortar, spatula, and powder paper), 7) receipts from PC and signature, 8) writing in the prescription 


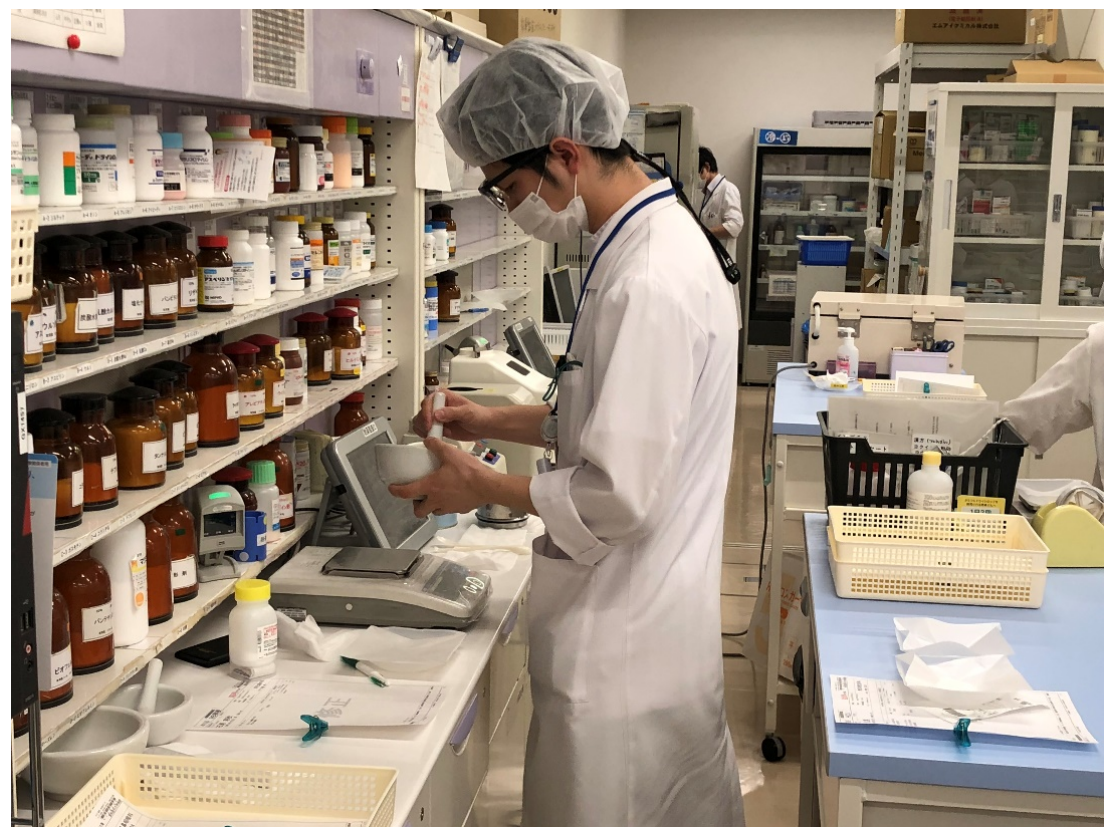

Figure 1. Tobii Pro Glasses 2.

(check marks and calculation of total amount), 9) mixing, and 10) others (writing materials, information sheets, etc.).

\subsection{Working Time for Each Behavior}

Actions within the whole work were divided into 7 categories. The categories were set as work, 1) preparation (cleaning on dispensing counter, preparation of dispensing apparatus, etc.), 2) checking prescription (inspection prescriptions, checking measured amount, writing in prescription), 3) weighing preparation (picking medication from the shelves, preparation of the powder paper and spatula, etc.), 4) weighing, 5) cleaning up (cleaning up dispensing counter and apparatus, etc.), 6) mixing, and 7) others.

\subsection{Statistical Analysis}

Differences in each parameter were statistically evaluated by the Student's t-test. All differences were considered significant when $p<0.05$.

\subsection{Ethics Statements}

This study is approved by the Kinjo Gakuin University Ethical Review Board (No. H17015).

\section{Result}

\subsection{Dispensing Time and the Content}

Table 1 shows the dispensing time of experts and newcomers. At ONE-P, the dispensing time of newcomer was significantly longer than expert $(p<0.05)$. Newcomer also tended to take longer time at TWO-P or TAB-P. In particular, 
dispensing time in "medication's shelf" of newcomer ( $9.4 \pm 6.0 \mathrm{sec}$.) was significantly longer than expert $(4.3 \pm 2.8 \mathrm{sec}).(p<0.05)$. It is suggested that newcomers took a long time to select medications from the shelves, because they did not remember the location of medications on the shelves.

For the work content, expert inspected the prescription and calculated the total amount before weighing. However, the actions of newcomer were started to weigh without inspection the prescription and simply relied on the powders' dispensing checking system. And, it is also showed insufficient preparations for weighing and no cleaning up on the dispensing counter. The dispensing process of newcomers lacked consistency compared with expert. Moreover, non-smooth dispensing process was also observed in certain newcomer.

\subsection{Gaze Measurement and Working Time for Each Behavior \\ 1) ONE-P}

The gaze time of medications of newcomer $(23.3 \pm 5.4 \mathrm{sec}$. $)$ was significantly longer than expert $(13.3 \pm 2.8 \mathrm{sec}$. $)(p<0.05)$ (Figure 2$)$. These results were suggested that newcomer took a longer time for looking many times from medications to weighing scale during the weighing process. Also, their gaze time of others was relatively longer. The gaze ratio per prescription was determined as the proportion of the gaze region time in whole working time. The gaze ratio of

Table 1. Dispensing time (sec).

\begin{tabular}{ccc}
\hline & expert & newcomer \\
\hline (1) ONE-P & $70.1 \pm 8.3$ & $104.4 \pm 19.9^{*}$ \\
(2) TWO-P & $141.3 \pm 8.0$ & $174.5 \pm 13.4$ \\
(3) TAB-P & $254.6 \pm 2.8$ & $416.8 \pm 94.6$ \\
\hline
\end{tabular}

mean $\pm \mathrm{SD}(\mathrm{n}=3-6),{ }^{*} p<0.05$.

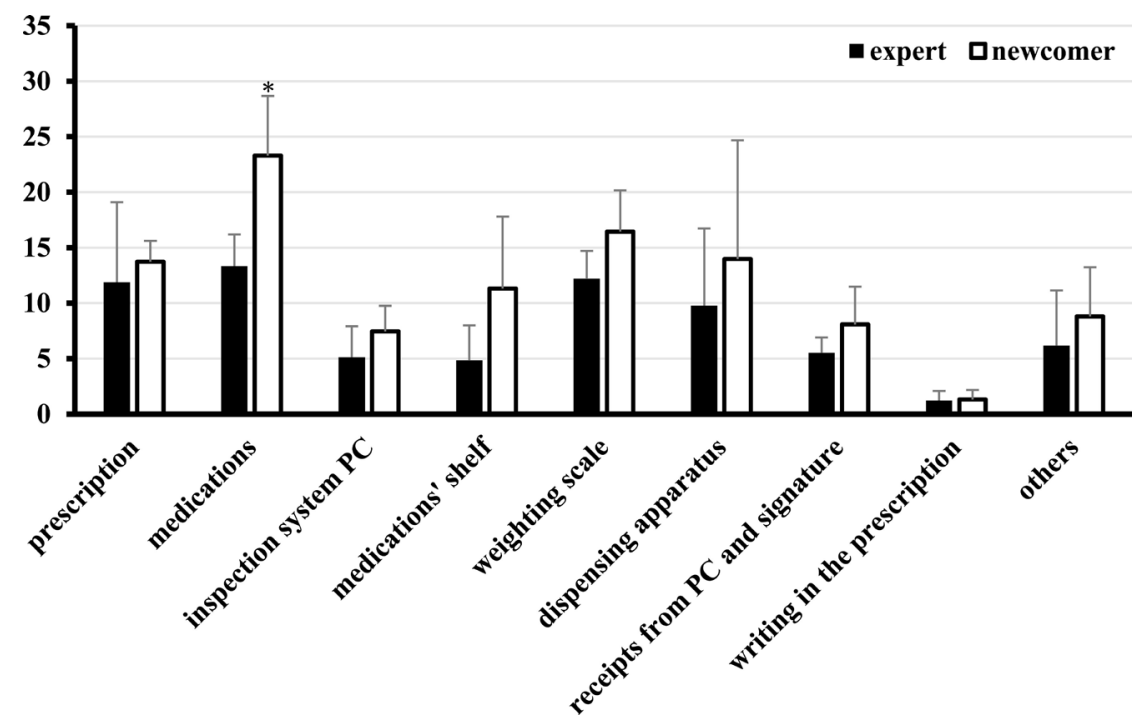

Figure 2. The gaze time at ONE-P(sec.) [mean $\left.\pm \mathrm{SD}(\mathrm{n}=6),{ }^{*} p<0.05\right]$. 
weighing scale of expert $(17.4 \% \pm 2.9 \%)$ was high, whereas that of medications of newcomer $(22.3 \% \pm 3.1 \%)$ was high. These results was suggested that experts concentrated on weighing scale's monitor during the weighing process, whereas newcomers alternately checked medications and weighing scale. The gaze ratio of prescription of expert $(16.9 \% \pm 9.1 \%)$ was higher in than newcomer $(13.3 \% \pm 1.7 \%)$.

Working time for each behavior of weighting of newcomer $(38.3 \pm 5.7 \mathrm{sec}$.) took significantly longer than expert $(23.4 \pm 4.5 \mathrm{sec}).(p<0.05)$. The behavior ratio of expert tended to be high about preparation $(7.5 \% \pm 5.5 \%)$, checking prescription $(8.1 \% \pm 5.5 \%)$ and cleaning up $(21.1 \% \pm 4.1 \%)$; therefore, these behaviors are important for experts. On the other hand, newcomer tended to be high about weighing preparation $(32.9 \% \pm 6.9 \%)$ and weighing $(37.2 \% \pm 5.1 \%)$.

2) TWO-P

The gaze time of inspection system PC of newcomer $(16.9 \pm 1.3 \mathrm{sec}$.) was significantly longer than expert $(8.9 \pm 2.0 \mathrm{sec}$. $)(p<0.05)$ (Figure 3$)$. The gaze time of mixing of newcomer $(20.6 \pm 1.8 \mathrm{sec}$.) was also longer than expert $(16.0 \pm 1.1$ sec.). Experts spent their time inspection of prescription and calculating to total amount before dispensing. On the other hand, newcomers dispensed to depend on inspection system PC without inspection of prescription. Therefore, their gaze time were longer for inspection system PC and shorter for writing in the prescription. The gaze ratio of inspection system PC of newcomer $(9.8 \% \pm 1.0 \%)$ was significantly higher than expert $(6.2 \% \pm 1.1 \%)$ like the gaze time $(p<0.05)$. The gaze ratio of writing in the prescription of expert $(4.2 \% \pm 1.0 \%)$ was higher than newcomer $(2.1 \% \pm 0.7 \%)$.

Working time for each behavior of weighting of newcomer $(61.0 \pm 16.2 \mathrm{sec}$.) took longer than expert ( $47.7 \pm 14.0 \mathrm{sec}$.) like at ONE-P. The behavior ratio of expert tended to be also high about checking prescription $(10.5 \% \pm 2.9 \%)$ and cleaning up $(13.9 \% \pm 1.2 \%)$, whereas newcomers focused on preparation $(6.6 \% \pm$ $2.2 \%)$, weighing $(36.0 \% \pm 11.2 \%)$, and mixing $(21.4 \% \pm 5.9 \%)$ like at ONE-P.

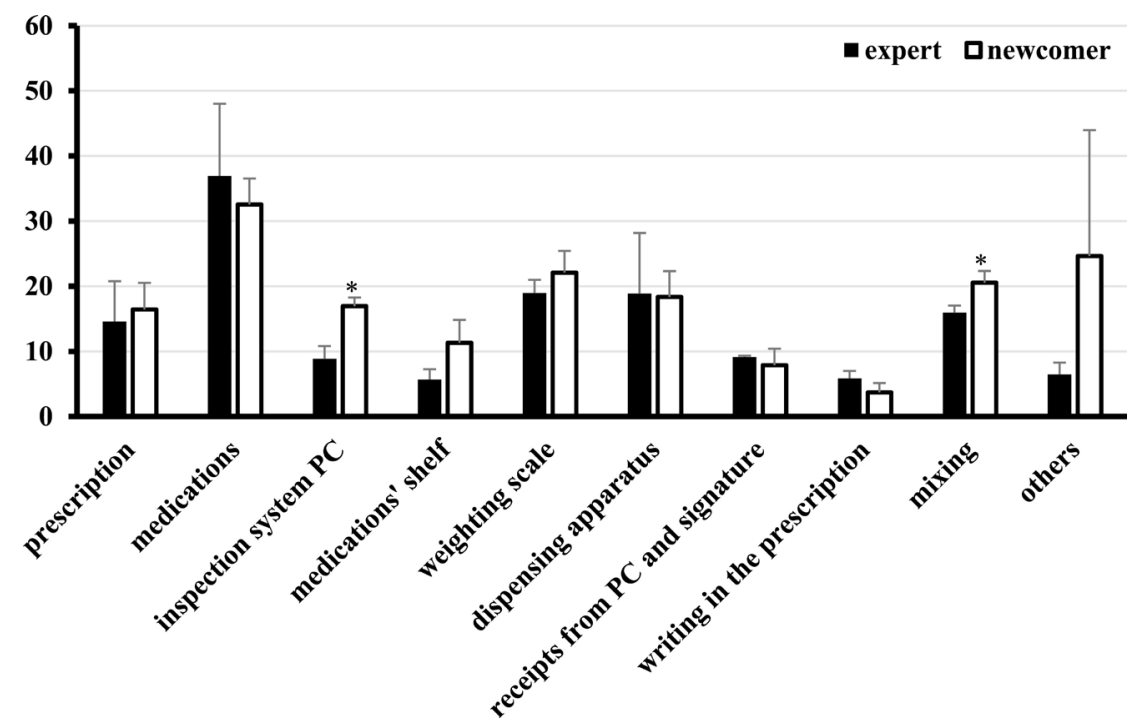

Figure 3. The gaze time at TWO-P (sec.) [mean $\left.\pm \mathrm{SD}(\mathrm{n}=3),{ }^{*} p<0.05\right]$. 


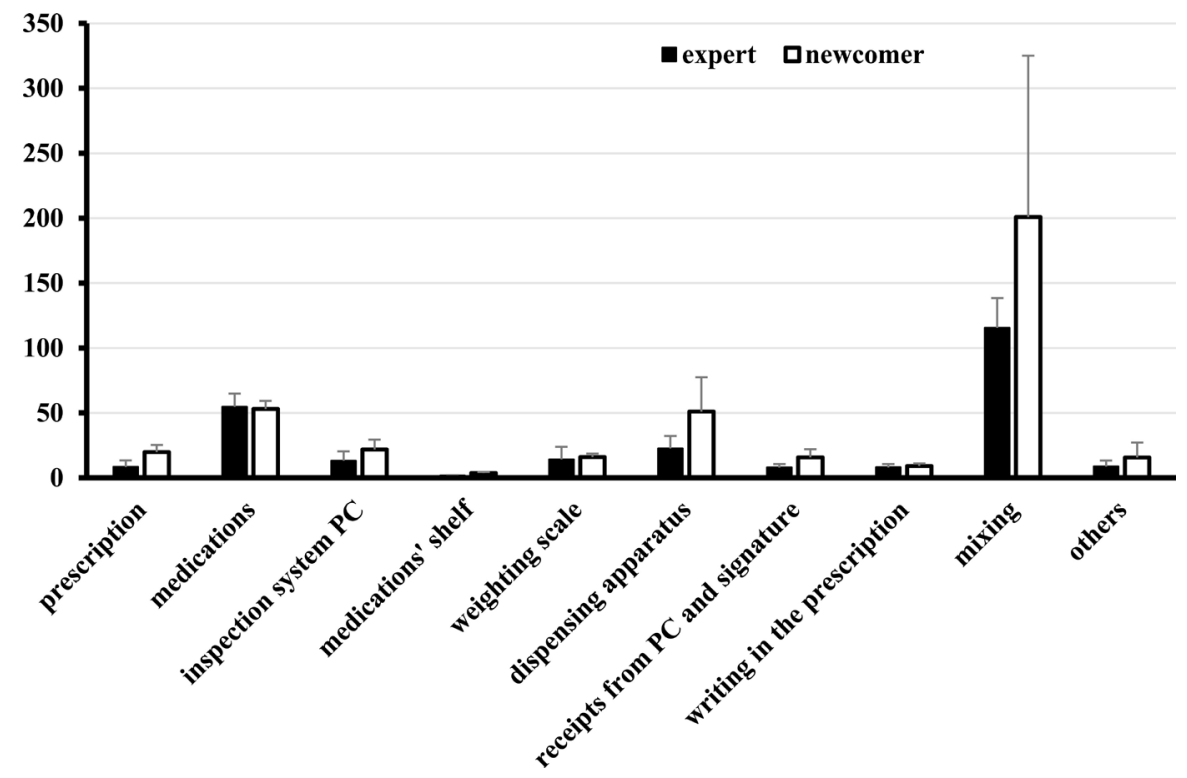

Figure 4. The gaze time at TAB-P (sec.) [mean $\pm \mathrm{SD}(\mathrm{n}=3),{ }^{\star} p<0.05$ ].

3) TAB-P

The gaze times of dispensing apparatus and mixing of newcomer were longer than expert (Figure 4). The gaze ratio of mixing was not significant difference between expert $(45.3 \% \pm 8.7 \%)$ and newcomer $(45.3 \% \pm 17.6 \%)$. These results were large standard errors, which reflected individual differences.

Newcomers tended to be longer behavior time in all divisions. Moreover, they were large standard errors of mixing and individual difference in work abilities was observed. Also, there was no significant difference between experts and newcomer about the behavior ratio.

\section{Discussion}

Approximately $70 \%$ of the near-miss events that occur at pharmacies relate to dispensing; the breakdown shows that "wrong quantity," "wrong specification/ dosage form," and "medication mix-up" are the most frequent with the Project to Collect Medical Near-miss/Adverse Event Information [1]. In this study, we studied to grope about the cause of dispensing errors at the powdered medication dispensing and investigate measures for preventing human error.

Based on the results of the eye tracking, newcomer had a longer dispensing time than expert for all powdered medication dispensing. In particular, it was suggested that there is a close relationship to "years of experience" and "weighing and mixing skills." Experts did unwasted and efficient movements, when preparing the dispensing apparatus, taking medications from the shelves, and scanning the barcode in the powders dispensing checking system. These movements led to shorter working time in experts. In contrast, newcomer had individual differences at the dispensing. Even with the same pharmacist, the work progression differed depending upon the prescription. Therefore, it is thought that the factors of common error were inadequate check and overlooked. The 
state that it's messy on the workplace, such as "insufficient preparations for weighing" and "leaving the medication bottles on the dispensing counter" is also considered highly likely to cause dispensing mistakes. At the weighing, expert started weighing after the inspection of the prescription and checking weighed amount. However, certain newcomer dispensed to depend on the powders dispensing checking system only for the weighing process, without the inspection of the prescription or checking weighed amount. Irregular doses for infants and older patients require fine adjustments; therefore, the dispensing checking system may not find all dispensing errors. It is important for a pharmacist to, first, be written calculated weight on the prescription and checked by themselves, and next to begin dispensation work. Therefore, the dispensing checking system should be used for double-checking.

In 2010, the Ministry of Health, Labour and Welfare advocated the following as regards methods of detailing information on oral medication prescriptions: "a prescribed dosage is single dose for oral medications rather than daily dose" and "a prescribed quantity is the product volume for powders and solutions rather than the active ingredient dosage" [8]. However, according to a 2015 survey, approximately $80 \%$ of the hospital stated only the daily dose; the practice is expected to shift gradually in the future. There is a possibility that a new error occurs by change in prescription mentioning method. It is necessary to consider unified methods of detailing information on prescriptions as an organization, in order to ensure that the content of the prescription intended by the physician is accurately conveyed to the pharmacist, and that everybody interprets the prescription in the same way. Therefore, it is suggested to have continual medical safety measures which considered medical staff's replacement at the educational and research organizations, such as university hospitals [9].

Additionally, the bottle of powder medications has similar packaging in terms of color, shape, and size. Sanbayashi et al. clarified that visual distinction can be identified most, when there is difference in an outward appearance package of medical supplies [10]. Therefore, similar medical supplies are also necessary for a prevention countermeasure of human error based on an environmental factor, such as making markings.

These results suggest that essential to have a smooth workflow from preparation for dispensing to weighing and mixing and to cleaning up, as well as to create a suitable working environment. The smooth work flow of the powdered medication dispensing as follows; 1) carry out the prescription inspection, 2) calculate the total amount to be weighed out, 3) check the powdered medication to be weighed out, 4) check the accuracy of the scales and weigh the powder, 5) if necessary, weigh out a lactose, 6) if there are multiple powders, mix them evenly, 7) divide the powders, 8) carry out an checking of the dispensed medication. In the future, as well as the powdered medication dispensing, it is necessary to make use of measures for preventing errors in the various dispensing process, such as the medication inspection, sterile products preparation, clinical practice et al. 


\section{Acknowledgements}

We would like to thank the pharmacy staff of the Department of Pharmacy, Aichi Medical University Hospital who cooperated in this study.

\section{Conflicts of Interest}

The authors declare no conflicts of interest regarding the publication of this paper.

\section{References}

[1] Division of Adverse Event Prevention of Japan Council for Quality Health Care (2017) Project to Collect Medical Near-Miss/Adverse Event Information. 2016 Annual Report, 28 August 2017.

[2] Haga, S. (2018) "Hyumanera no rinri to taisaku." NST Inc., Japan. (In Japanese)

[3] Kawano, R. (2018) "Taisaku ha ri ni kanaukoto! hyu-mannerataisaku no kanngaekata." Journal of the Japan Pharmaceutical Association, 70, 839-842. (In Japanese)

[4] Sekine, Y., Mitsuyama, H. and Hosoda, J. (2003) "Sanzaikeiryouchouzai ni okeru risukumanejimento no kokoromi." Medication and Drug Journal, 39, 147-152. (In Japanese)

[5] Division of Adverse Event Prevention of Japan Council for Quality Health Care (2010) Project to Collect Medical Near-Miss/Adverse Event Information. 24 October-December 2010.

[6] Tobii Technology K.K. (2018) https://www.tobiipro.com/ja/

[7] Miyachi, Y., Nakayama, C., Nagashiba, K., Kinoshita, K., Takeuchi, M., Ohnishi, M., Saito, H. and Oshima, T. (2018) Study of Factors Affecting Medical Incident: 1. Dispensing. Pharmacology \& Pharmacy, 9, 527-535.

https://doi.org/10.4236/pp.2018.912041

[8] Ministry of Health, Labour and Welfare (2010) Report of the Panel on Approaches to Methods of Detailing Information on Oral Drug Prescriptions. January 2010. http://www.mhlw.go.jp/shingi/2010/01/dl/s0129-4a.pdf

[9] Ogawa, K., Adachi, M., Wakushima, H., Kaneda, T., Shiinoki, Y., Shimada, M., et al. (2017) Implementation of a Standardized Method of Writing Prescriptions for Oral Medication and Evaluation of the Medical Safety Management. Journal of Japanese Society of Hospital Pharmacists, 53, 560-566.

[10] Sanbayashi, Y., Tsuchiya, F., Takenouchi, T. and Murayama, J. (2008) Line-of-Sight Measurement and Human Errors during Drug Preparation. IEICE Technical Report SSS, 108, 9-12. 